\title{
Achievement of ultrahigh quality factors in GaAs photonic crystal membrane nanocavity
}

\author{
Evelin Weidner, ${ }^{\text {a) }}$ Sylvain Combrié, Nguyen-Vi-Quynh Tran, Alfredo De Rossi, \\ Julien Nagle, and Simone Cassette \\ Thales Research \& Technology, Route Départementale 128, 91767 Palaiseau, France
}

Anne Talneau

Laboratoire de Photonique et Nanostructures, Route de Nozay, 91460 Marcoussis, France

Henri Benisty

Laboratoire Charles Fabry de l'Institut d'Optique, Bât. 503, 91403 Orsay, France

(Received 2 August 2006; accepted 5 October 2006; published online 27 November 2006)

\begin{abstract}
The authors realized an ultrahigh quality factor nanocavity in a GaAs membrane with the highest loaded $Q$ reported to date of 250000 in a side-coupled cavity-waveguide system. This result could be obtained using an original aluminum-free material system combined with a carefully adjusted fabrication technology, yielding a device with small roughness and very good verticality of holes as well as small disorder. The authors show that the intrinsic $Q$ factor is around $3.0 \times 10^{5}$ using a coupled-mode model. (C) 2006 American Institute of Physics. [DOI: 10.1063/1.2390648]
\end{abstract}

Optical microcavities of high quality factors in twodimensional (2D) photonic crystals (PCs) are known to provide effective field confinement [of the order of $(\lambda / n)^{3}$ ], resulting in very high Purcell factors, which makes them suitable candidates, in particular, for cavity quantum electrodynamics (QED), ${ }^{1,2}$ but also for nonlinear optics applications in photonics and telecommunication as the required pump powers can be very weak. ${ }^{3,4}$ The recent theoretical demonstration of all-optical flip-flop circuits ${ }^{5}$ provides a striking example.

Important progress has been made in the domain of $2 \mathrm{D}$ PC microcavities, principally due to the use of membrane technology and the understanding of loss mechanisms. Very recently, ultra-high- $Q\left(10^{6}\right)$ single-mode microcavities have been demonstrated based on Si membrane PCs. ${ }^{6,7}$ GaAsbased microcavities are indeed attractive for QED due to the excellent optical properties of this material and, in particular, the quality of quantum dots that can be grown on it. However, the performances of GaAs-based microcavities have not reached those of their Si-based counterparts. The highest $Q$ factor obtained in GaAs is of the order of $140000 .^{8}$ The physical mechanisms that limit the $Q$ factor of GaAs microcavities have not been elucidated yet, neither the maximum $Q$ that can be achieved.

The 2D PC membrane microcavity presented here consists of a double heterostructure following the design principle recently proposed by Song et al. ${ }^{6}$ Light confinement is obtained by locally changing along one crystal direction the lattice period of a waveguide section from $a_{1}$ $=410 \mathrm{~nm}$ to $a_{2}=420 \mathrm{~nm}$, as shown in Fig. 1(a), resulting in an effective photonic potential well. This design has already been shown to provide very high quality factors both in $\mathrm{Si}$ and in GaAs, as mentioned before. Thanks to an original fabrication technology, ${ }^{9}$ based on the use of a GaInP sacrificial layer and a high density plasma etching process, ${ }^{10}$ we show that for this cavity design, the $Q$ factor in GaAs membranes can be greatly improved.

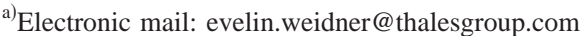

The various fabrication steps include e-beam lithography and reactive ion etching to define the mask design. This is followed by a chlorinated inductively coupled plasma etching process to transfer the pattern into the semiconductor, and $\mathrm{HCl}$ wet etching to remove the GaInP sacrificial layer and to realize the structured GaAs membrane. GaInP is lattice matched to GaAs and assures high etching selectivity in the last step thanks to the large compositional difference between anions. ${ }^{10}$ The whole process has been carefully optimized in order to minimize disorder and roughness at each fabrication stage and to guarantee a good verticality of the hole walls and very smooth membrane top and bottom surfaces [Figs. 1(b) and 1(c)].

For our sample, the membrane is $250 \mathrm{~nm}$ thick and the nominal hole radius is $r=0.3 a_{1}$. A line-defect waveguide formed by one missing row of holes is in the $\Gamma K$ direction, forming the cavity together with the double heterostructure. The investigation of such a microcavity requires the coupling of light via another side-coupled waveguide. In our case, the waveguide-to-cavity separation is six rows of holes in order

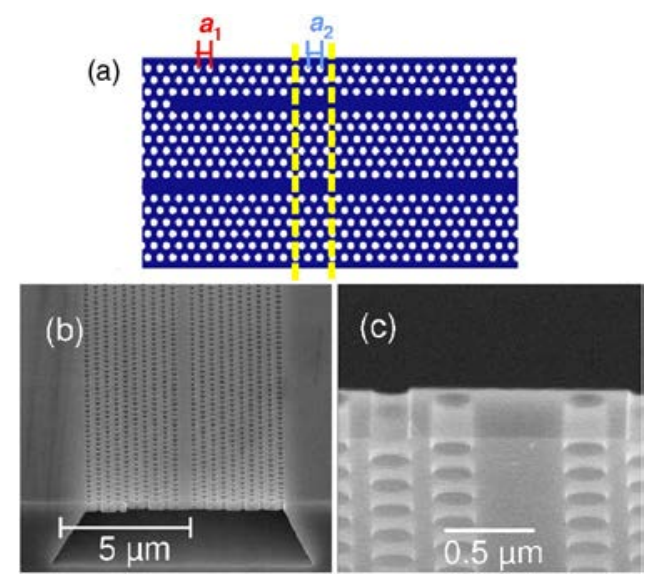

FIG. 1. (Color online) (a) Schematic description of the side-coupled cavity system. (b) Scanning electron microscope image of the entrance face of the PC input waveguide. (c) Holes seen by transparency through the GaAs membrane. 


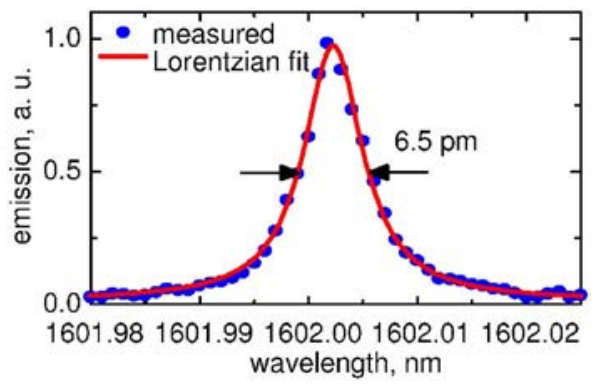

FIG. 2. (Color online) Detailed vertical emission spectrum of the nanocavity near the resonant wavelength.

to adjust the extraction efficiency. This waveguide is $0.14 a_{1}$ wider than the other one.

The transmission spectrum of the coupled system as well as the vertical emission spectrum of the cavity were measured using a tunable external cavity laser diode, with $1 \mathrm{pm}$ spectral resolution (Nettest) as the light source. The light is coupled into the waveguide and collected after transmission through the sample by two microscope objectives. A third microscope objective is used to image a tiny region around the cavity and collect its vertically scattered radiation. A typical vertical emission spectrum around the cavity resonance is presented in Fig. 2. We estimated the linewidth of the narrow emission peak using a Lorentzian fit and found a value of $6.5 \mathrm{pm}$, corresponding to a loaded quality factor of 246000 . This is the highest value reported in any wavelength-sized GaAs-based microcavity to date. In the following, we analyze the coupling between the waveguide and the cavity in order to estimate the intrinsic $Q$ factor. We use a simple coupled-mode theory model to describe the side-coupled microcavity-waveguide structure with partially reflecting elements in the waveguide. ${ }^{11,12}$

Measurements of the waveguide transmission and the cavity vertical emission as a function of the wavelength were carried out simultaneously. Characteristic spectra in the vicinity of the cavity resonance are shown in Fig. 3. We indeed observe a sharp and asymmetric lineshape in the transmission response. It consists of a resonant feature superimposed upon a background defined by the Fabry-Pérot (FP) oscilla-

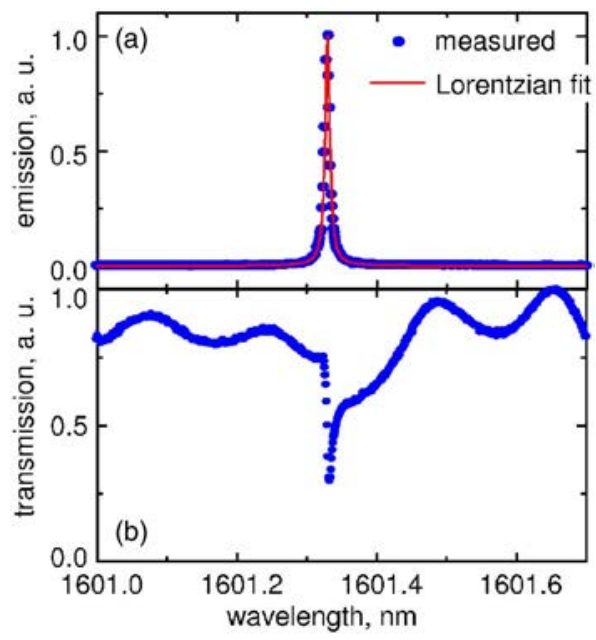

FIG. 3. (Color online) (a) Emission spectrum of the nanocavity near the resonance. The full width at half maximum is estimated using a Lorentzian fit; (b) corresponding transmission spectrum of the waveguide with the drop caused by the cavity. It is due to the carefully adapted fabrication process that
Downloaded 04 Dec 2006 to 129.104.38.4. Redistribution subject to AIP license or copyright, see http://apl.aip.org/apl/copyright.jsp

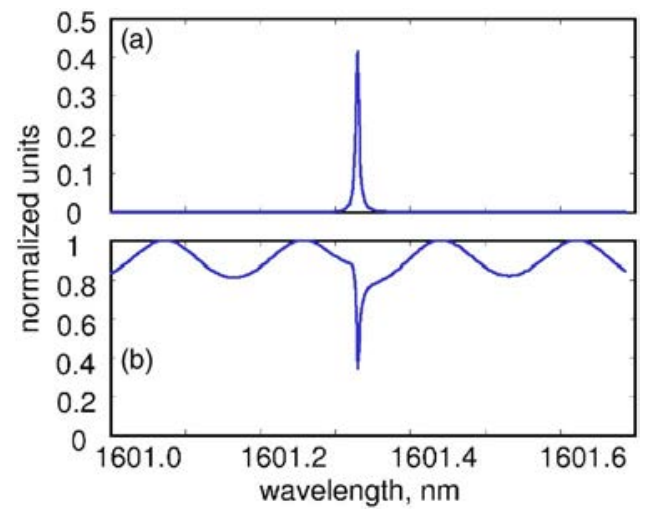

FIG. 4. (Color online) Calculated vertical emmission (a) and transmission (b) spectra near the cavity resonance, using the experimental parameters.

tions. The waveguide terminations actually act as partially reflecting elements that lead to interference phenomena through the modified phase of the reflected waves. Together with a group index of $n_{g} \approx 7$, the FP fringe spacing indicates that these oscillations are indeed related to the device length of $1 \mathrm{~mm}$. As can be seen in Fig. 3(b), the cavity resonance is situated at the onset of a rising edge of a FP fringe, and the dip in the transmission shows an asymmetric form. Note that the experiments were carried out at very low powers (estimated $<10^{-5} \mathrm{~W}$ coupled in the waveguide) so that the presence of optical nonlinear effects can be excluded.

We normalized the maximum FP fringe signal at unity away from resonance. The system transmission coefficient varies sharply from $76 \%$ to $30 \%$ close to the resonance frequency at the shorter wavelength side, which means that a transmitted signal attenuationof more than $60 \%$ is obtained. It does not drop to zero because of cavity losses. While the interpretation of the vertical emission spectrum is straightforward, the quantitative analysis of the transmission response is more involved. We adopted the T-matrix formalism described in Ref. 9 in order to account for the observed behavior. It is important to separate the waveguide-to-cavity coupling, $\gamma$, from the intrinsic cavity linewidth $\Gamma_{0}$. The latter is represented by $\Gamma_{0}=\omega_{0} / Q_{\text {in }}$, so that we can write $\Gamma=\Gamma_{0}+\gamma$ $=\omega_{0} / Q$, where $\gamma=\omega_{0} / Q_{c}$. Here, $Q, Q_{\text {in }}$, and $Q_{c}$ are the total, intrinsic, and coupling quality factors of the cavity, respectively. The resulting transmission of the coupled system and the cavity out-of-plane emission are depicted in Fig. 4. With that, we found a coupling of $Q_{c} \approx 1.2 \times 10^{6}$, which is consistent with our three-dimensional finite difference time domain modeling carried out simultaneously. The unloaded qualtity factor of the cavity is estimated to be about $3 \times 10^{5}$.

Such high- $Q$ cavities coupled to finite-length singlemode waveguides have recently been theoretically shown to induce sharp resonant features in the response characteristic of the whole device. ${ }^{12}$ In contrast to our case, the cavity in Ref. 12 was considered to have an infinite intrinsic quality factor. The relative spectral position of the resonance with respect to the FP fringes and hence its form can be adjusted by controlling the exact value of the cavity resonance frequency.

In conclusion, we have reported on obtaining an ultrahigh $Q$ factor in a GaAs membrane-based doubleheterostructure nanocavity. The loaded value was almost 250000 , which is the highest reported value to date in GaAs. 
ensures minimal disorder and very good hole wall verticality. These GaAs high- $Q$ cavities are well-suited structures to study (i) QED phenomena (strong coupling) as state-of-theart quantum dots can be integrated on it, and (ii) nonlinear optical effects (leading to optical bistability) as light is very strongly confined. On the other hand, the cavity, side coupled to a PC waveguide, generates sharp and asymmetric line shapes in the transmission spectrum of the system. This can be exploited for several applications such as filtering or switching. Furthermore, we could identify a coupling quality factor of over $10^{6}$, which suggests that an energy management at the nanosecond scale can be achieved thanks to proper technology.

This work study was partly funded by the European Commission within the project QPhoton under Contract No. IST-029283. One of the authors (E.W.) acknowledges the allocation of the EU Descartes Prize, given to the ISTQuComm Project in 2004, as a postdoctoral grant.
${ }^{1}$ T. Yoshie, A. Scherer, J. Hendrickson, G. Khitrova, H. M. Gibbs, G. Rupper, C. Ell, O. B. Shchekin, and D. G. Deppe, Nature (London) 432, 200 (2004).

${ }^{2}$ A. Badolato, K. Hennessy, M. Atatüre, J. Dreiser, E. Hu, P. M. Petroff, and A. Imamoğlu, Science 308, 1158 (2005).

${ }^{3}$ P. E. Barclay, K. Srinivasan, and O. Painter, Opt. Express 13, 801 (2005).

${ }^{4}$ T. Uesugi, B. S. Song, T. Asano, and S. Noda, Opt. Express 14, 377 (2006).

${ }^{5}$ A. Shinya, S. Mitsugi, T. Tanabe, M. Notomi, I. Yokohama, H. Takara, and S. Kawanishi, Opt. Express 14, 1230 (2006).

${ }^{6}$ B. S. Song, S. Noda, T. Asano, and Y. Akahane, Nat. Mater. 4, 207 (2005).

${ }^{7}$ E. Kuramochi, M. Notomi, S. Mitsugi, A. Shinya, T. Tanabe, and T. Watanabe, Appl. Phys. Lett. 88, 041112 (2006).

${ }^{8}$ R. Herrmann, T. Sünner, T. Hein, A. Löffler, M. Kamp, and A. Forchel, Opt. Lett. 31, 1229 (2006).

${ }^{9}$ S. Combrié, E. Weidner, A. De Rossi, S. Bansropun, S. Cassette, A. Talneau, and H. Benisty, Opt. Express 14, 7353 (2006).

${ }^{10}$ S. Combrié, S. Bansropun, M. Lecomte, O. Parillaud, S. Cassette, H. Benisty, and J. Nagle, J. Vac. Sci. Technol. B 23, 1521 (2005).

${ }^{11}$ H. A. Haus and Y. Lai, J. Lightwave Technol. 9, 754 (1991).

${ }^{12}$ S. Fan, Appl. Phys. Lett. 80, 908 (2002). 